\title{
Switching to teleconsultation for rheumatology in the wake of the COVID-19 pandemic: feasibility and patient response in India
}

\author{
Padmanabha Shenoy $^{1}$ (D) $\cdot$ Sakir Ahmed ${ }^{2}$ (D) $\cdot$ Aby Paul $^{1} \cdot$ Teny Grace Skaria $^{1} \cdot$ Joel Joby $^{1} \cdot$ Bazil Alias $^{1}$
}

Received: 24 April 2020 / Revised: 19 May 2020 / Accepted: 22 May 2020 / Published online: 30 May 2020

(C) International League of Associations for Rheumatology (ILAR) 2020

\begin{abstract}
The emergent COVID-19 pandemic dictates an urgent switch to teleconsultation. India has high patient to rheumatologist ratio, and patients have limited concepts about telemedicine. Thus, we attempted to find the feasibility and acceptance of patients in switching to teleconsultation. The CARE rheumatology clinic at Kerala, India, caters to average 170 (range: 140-240) patients per day. Patients with prefixed appointments had two-level screening for eligibility for teleconsultation. Those eligible were given the option for teleconsultation on the widely available WhatsApp app. Of those who completed teleconsultations, 100 were chosen at random to provide feedback. In the first 7 days, out of 1469 appointments, 975 were found eligible for teleconsultation. Of these, 723 (74\%) opted for it. The average footfall in the clinic was reduced to 67 (range 29-117). The proportion of patients accepting teleconsultations increased with time. Amongst the 100 respondents, median satisfaction was 9 (IQR 8-10) and recommendation for continuing was 9.5 (IQR 8-10) on a 0-10 scale. Multivariate analysis showed the recommendation score was dependent on beliefs about social distancing, perceptions about clinical examination, and the satisfaction score of the first teleconsultation. Age, sex, availability of personal video conferencing app or of vehicles did not independently influence this score. Without teleconsultation facilities, three-fourths of the respondents would have stopped drugs or self-medicated. The switch was feasible and accepted by patients. It enabled quick reduction in the number of persons travelling to the centre. Not making the switch could have deprived approximately three-quarters of these patients of proper medical care.
\end{abstract}

\section{Key Points}

- Patient to rheumatologist ratios in India is heavily skewed and awareness about telemedicine is limited.

- Switch to telemedicine was feasible and allowed a decrease in the number of people attending the clinic.

- Not switching could have lead to disruption of care or self-medication in a majority of patients.

Keywords COVID-19 $\cdot$ Telemedicine $\cdot$ Telerheumatology $\cdot$ Social distancing $\cdot$ Video consultation

Padmanabha Shenoy and Sakir Ahmed share joint first authorship

The corresponding author attests that all listed authors meet authorship criteria and that no others meeting the criteria have been omitted.

Electronic supplementary material The online version of this article (https://doi.org/10.1007/s10067-020-05200-6) contains supplementary material, which is available to authorized users.

Padmanabha Shenoy

drdpshenoy@gmail.com

Sakir Ahmed

sakir005@gmail.com; sakir.ahmed@kims.ac.in

Aby Paul

26abypaul@gmail.com

Teny Grace Skaria

teny18gs@gmail.com
Joel Joby

joeljoby777@gmail.com

Bazil Alias

bazilaliaskuttuvelil@gmail.com

1 Centre for Arthritis and Rheumatism Excellence (CARE), Nettoor, Cochin, India

2 Department of Clinical Immunology \& Rheumatology, Kalinga Institute of Medical Sciences (KIMS), KIIT University, Bhubaneswar 751024, India 


\section{Introduction}

The novel coronavirus disease (COVID-19) first reported from Wuhan, China, was declared a pandemic by the World Health Organization on the 11th of March, 2020 [1]. India's first cases to be identified as positive by the 30th of January, 2020 were three students from Kerala (Indian state) who had travelled from Wuhan [2]. As of the 22nd of March, the number of cases in India and Kerala had reached 360 (including 41 foreign nationals) and 67, respectively [3]. The total population of Kerala as per 2011 census was 33,406,061, and the population density was 860 per square km [4]. Kerala had been recently successful in controlling the outbreak of Nipah virus in 2018 [5]. Armed with this previous experience, the state had sprung early into action with screening of foreign travellers and extensive contact tracing.

It became imperative to control patient flow to hospitals to minimise spread. However, medical services to chronic patients, like those in rheumatology, needed to go on uninterrupted. In India, the patient to rheumatologist ratio is highly skewed with rheumatology centres dealing with large volume of patients [6].

Switching to teleconsultation was a practical strategy. However, the country has limited experience with telemedicine. Certain patients may not be exposed to concepts of video calling, far less video consultation. However, India has high prevalence of smartphone usage, with more than 340 million users of the WhatsApp app. Since this app has video calling facility, we determined to use it as a means for teleconsultation. The feasibility and patient acceptance of such an approach needed to be explored. This was initiated from the 12th of March, 2020, and after seven working days till the 20th of March, we carried out an audit of the switch and took feedback from patients.

\section{Methods}

The study was an audit of the feasibility and utility of switching to teleconsultation from in-person visits. The switch was attempted at a stand-alone rheumatology clinic, CARE (Centre for Arthritis and Rheumatism Excellence), at Kerala, India. CARE caters to average of 170 patients daily from all districts of Kerala. Kerala is the southern state of India with a length $580 \mathrm{~km}$. CARE as situated in the middle part of Kerala, we had patients travelling up to $250 \mathrm{~km}$ to reach the clinic, some of them using public transport.

Rheumatology patients who had prefixed appointments were considered for teleconsultation if they did not have any fresh complaints in the previous visit and could get their blood tests done at local laboratories. Patients who had been planned for any procedure (intravenous infusion of biologicals or intraarticular injection), for any diagnostic tests (imaging or interventional) or who had to procure subcutaneous biological injections were excluded. Patients who had not completed at least two visits or had any diagnostic dilemma were also excluded. First screening was done via electronic medical records (EMR).

The patients eligible on the first screening were contacted by appointment staff, who interviewed the patient regarding symptoms of disease flare as per fixed protocol (Figs. 1 and 2). If any patient had any new symptoms on the second (telephonic) screening, they were suggested personal visits. All others were informed about the importance of preventing crowding in the clinic and reducing social movement. Then, they were offered either teleconsultation or personal visit or rescheduling their appointment. The patients opting for teleconsultation were asked about the availability of smart phone and familiarity with video calling feature of WhatsApp. The patients who agreed for teleconsultation were instructed to get required tests done at local laboratories.

On the date of the teleconsultation appointment, patients uploaded their test reports using WhatsApp along with a picture of their registration card to avoid errors. The teleconsultation was started on WhatsApp video by a junior doctor who updated the EMR. The patients were encouraged to focus the camera on previous skin rash, joint swelling and other visible manifestations. Joint movements were visualised as required. This was followed by consultation by a rheumatologist who then finalised medication. The prescribed medicines were packed by the pharmacy and dispatched by courier once billing formalities had been completed.

After seven working days of switching to online consultations, we conducted an audit of the system to see its feasibility and acceptance. Patients who had attended teleconsultation called up at random until feedback from at least 100 had been received. Feedback included eight questions. Two questions were to be rated on a Numeric Rating Scale (NRS) of 0 to 10, and rest had two or more fixed options (Supplementary Table 1).

Data analysis: A descriptive analysis of the audit and survey is being presented. To explore if rising number of COVID-19 was influencing people's decision to opt for teleconsultation, a correlation was calculated with the number of COVID-19 cases reported in Kerala on each day with the number of patients opting for teleconsultation. We hypothesised that the following factors would influence the decision of patients to continue with teleconsultation: age, sex, beliefs about social distancing, satisfaction in first teleconsultation, apprehension of missing physical examination, dependence on others for use of WhatsApp or availability of private vehicle. Using generalized linear models, a multivariate analysis was carried out using these factors as covariates.

Since this was a quality assurance activity, it falls outside the criteria necessary for ethics review. 
Fig. 1 Schematic showing the protocol for screening of patients for eligibility for teleconsultation

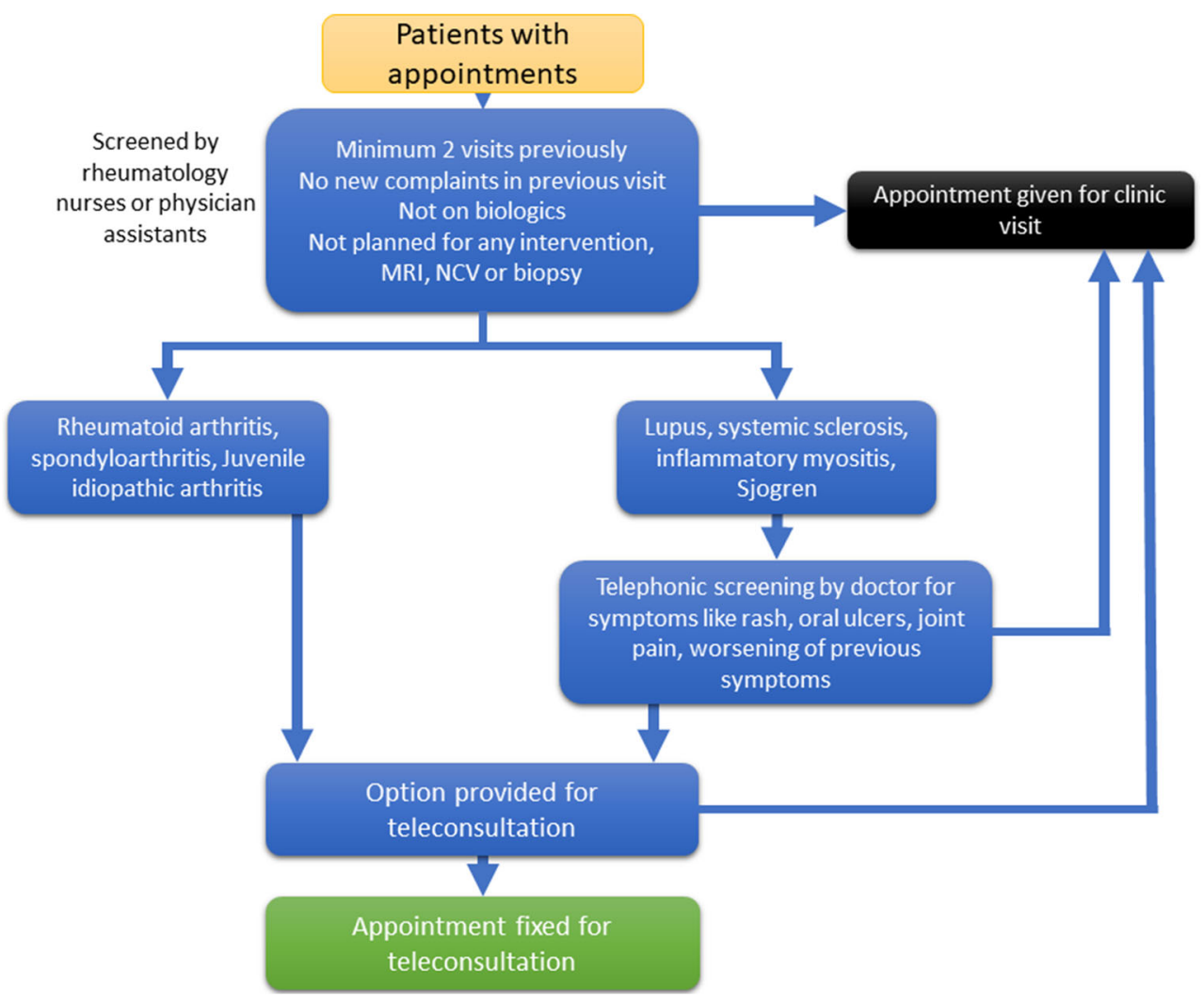

\section{Results}

In the 7 days, 975 out of 1469 appointments were offered teleconsultation of which 723 (74\%) agreed, while a total of 275 cancelled or rescheduled appointments. The average number of cancelled appointments rose from 25 to 39 per day (56\%). Compared with the previous 6-month average of 170 (range 140-240) outpatient visits daily, these 7 days had
67.3 (range 29-117) outpatient visits daily (Fig. 1). A moderate positive correlation $\left(r^{2}=0.67, p=0.025\right)$ existed between the proportion of patients opting for teleconsultation and the total number of COVID-19 cases reported in Kerala [3] on that same day, that is from 12th to 20th of March, 2020.

One hundred forty nine patients were called once each until the criterion of feedback from 100 was reached. Median age was 54 (IQR 44.25-64) and 87 were females. Overall median
Fig. 2 Proportions of patients found eligible for and attending teleconsultation as well as proportion cancelling appointments

\section{Proportion of patients attending teleconsultation}

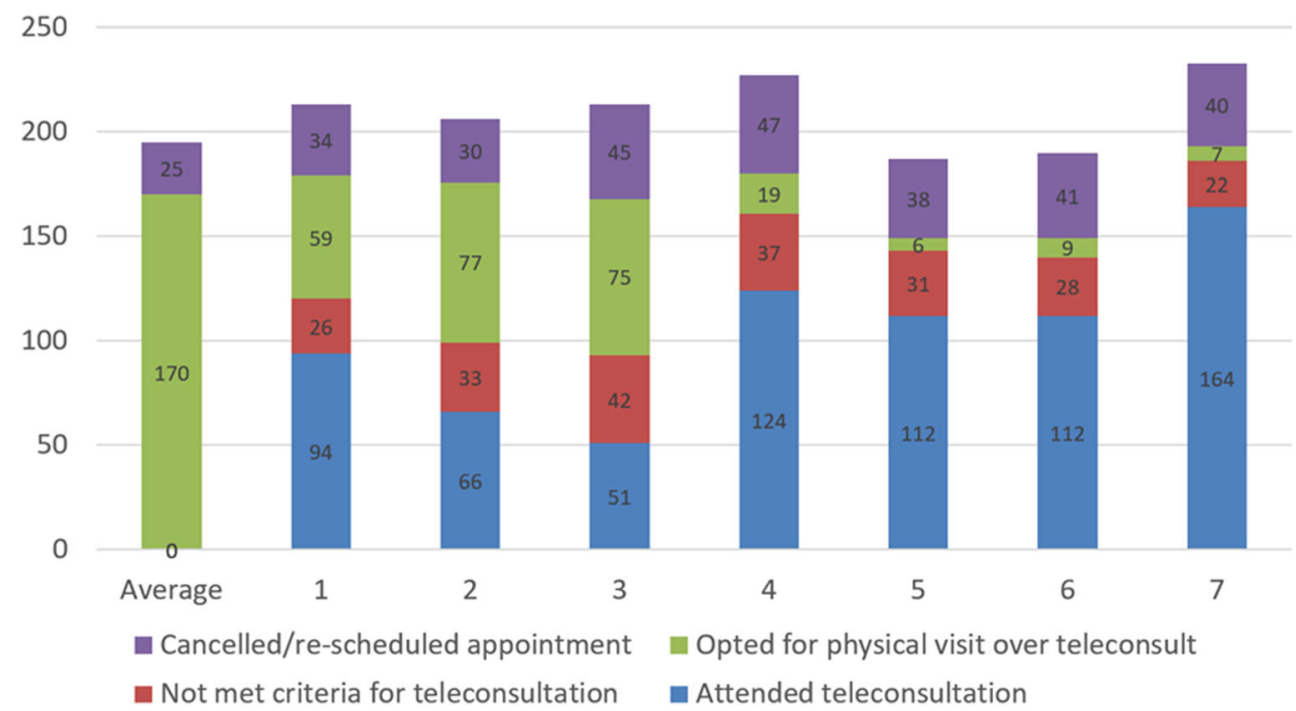


satisfaction was 9 (IQR 8-10) on the NRS scale. The respondents scored median 9.5 (IQR 8-10) on the NRS scale in recommending the continuation of teleconsultation. Under normal conditions, each patient would have brought a median 1 (range 1-2) accompanying persons. The responses for other variables are summarized in Table 1.

A multivariate analysis using generalized linear model showed that the agreement for continuing teleconsultation depended on beliefs that social distancing would reduce the pandemic, that the doctor might have missed something without physical examination and on the satisfaction with the online consultation. Non-significant variables in the multivariate model were age, sex, dependence on others for use of WhatsApp or availability of private vehicle.

\section{Discussion}

This study has looked at three aspects: first, the feasibility of carrying out video consultations in India using a commonly available mobile app; second, its impact on reducing the number of patients visiting the clinic and third, the patient satisfaction and acceptance of teleconsultation.

Regarding the feasibility, WhatsApp was chosen as it is the leading social media platform in India with more than 340 million users [7]. Around $50 \%$ of patients did not have the app on their own phones or were not acquainted with its use. However, they could enlist the help of their relatives or friends and thus attend the video conferencing. Video conferencing may be taken for granted in certain countries or even in certain societies in India. However, its use of medical purposes across all strata of society was a social experiment. The question on feasibility was measured by the other two aspects of the study: it helped to decrease the number of people coming to the clinic and also had good patient satisfaction.
The switch reduced the number of patient visits by $60 \%$ in the first 7 days. This proportion of patients opting for teleconsultation was increasing daily (Fig. 1). On the last 2 days, more than $75 \%$ were using teleconsultation facilities. Amongst the persons eligible for teleconsultation, the number of persons opting out was in single digits in the last 3 days. With 723 teleconsultations, approximately 1590 (227 per day) less persons had to come to the clinic.

An average of 31 patients per day did not meet our criteria for teleconsultation. These were patients who had unsolved problem in previous visits, new symptoms at present or some dilemma in diagnosis. Thus, quality of care was not compromised by switching to teleconsultation for every patient. The average number of cancelled appointments rose from 25 to 39 per day $(56 \%)$. A majority of these patients postponed appointments (data not shown), possibly in the hope that the COVID-19 crisis will soon tide over. It should be kept in mind that this switch took place just when WHO declared the pandemic, and India was starting to plan accordingly.

The implications of reduction of patient numbers are important. Different countries have different health care capabilities to combat COVID-19 [8], and rapid community spread though India can easily overwhelm the existing healthcare system. The secondary impact of the pandemic may be increased mortality due to other infectious disease and malnutrition like seen in Africa after the Ebola outbreak [9]. Thus, it is imperative to stem the pandemic in India as early as possible.

The third and most important part relates to patient acceptance. Acceptance was rated 9/10 while recommendations for continuing this system were scored at 9.5/10. Though around one-fifth of the respondents felt uncomfortable without a physical consultation, more than two-thirds were alright with it.

Patient acceptance is the most important to predict sustainability. This was also seen in the multivariate analysis.
Table 1 Qualitative variables assessed during postteleconsultation survey

\begin{tabular}{ll}
\hline Variable & Frequency \\
\hline Felt that doctor missed something important in video consultation & Definitely 5 \\
& Maybe 17 \\
& Not sure 10 \\
& Unlikely 11 \\
Believed that social distancing will help reduce Pandemic & No 57 \\
Depended on others for use of WhatsApp video conferencing & 92 \\
Availability of personal vehicle & 51 \\
Alternative course had telemedicine not been available & 46 \\
& Come to clinic 26 \\
& Stop medicine 44 \\
& Self-medicate 30 \\
\hline
\end{tabular}


Patients who had higher satisfaction scores were more likely to recommend continuation, irrespective of age, gender or even availability of video conferencing on their own phones.

The use of teleconsultation in India has been limited. This may be due to absence of guidelines and of legal perspective regarding telemedicine in the country [10]. Telemedicine has been advocated mostly as a bridge to primary care in far-flung regions or in the defence services [11]. A systematic review has found it useful for both diagnosis and management, but based on limited evidence [12]. Telemedicine had been predicted to take up a major chunk of rheumatology consultations in the near future [13]. We believe that the COVID-19 pandemic has only hastened this switch. This is supported by the fact that as the number of COVID-19 patients from Kerala increased, the proportion opting for teleconsultation increased. This is apparently due to people understanding the implications of the pandemic. In previous outbreaks, it has been shown that fear is a factor influencing social distancing behaviour [14].

In the post-teleconsultation survey, around one-quarter said they would have attended the clinic if no other option was available. This left a very high number who would have either stopped the drugs or self-medicated, both having their own dangers. Medicine misuse is widespread in India [15], and this can easily be compounded. This indicates the importance of the switch at this time.

One limitation of the study is the lack of follow-up. However, the aim of the current audit was to understand the immediate feasibility and patient reactions. Follow-up can be planned to compare outcome patients who opted for teleconferencing with those who did not. The second limitation can be the lack of generalizability to other parts of the country where experience of telemedicine may be limited. Again, our experience was also limited and we depended on freely available social media platforms like WhatsApp. India had 502.2 million smartphone users as of December 2019 [16], and this makes the use of smartphones the ideal platform for teleconsultation even in distal places where other forms of electronic communication are limited.

In conclusion, this switch was feasible and well-accepted by patients, and it enabled quick reduction in the number of persons travelling to the centre. Not making the switch could have deprived approximately three-quarters of these patients of proper medical care, leading to self-medication or stopping of their drugs.

Author contributions The conception and design of the study: SA, PS. Acquisition of data: TGS, AP, JJ, BA. Analysis and interpretation of data-SA, AP, TGS.

Drafting the article - SA, PS. Revising it critically for important intellectual content-PS, TGS, AP, JJ, BA.

Final approval of the version to be submitted - all four.

Agreement to be accountable for all aspects of the work in ensuring that questions related to the accuracy or integrity of any part of the work are appropriately investigated and resolved - all four.
Data availability Data will be shared by corresponding author on reasonable request.

\section{Compliance with ethical standards}

Disclosures None.

Ethical approval This article is based on the results of an audit, and being a quality assurance service, does not quality for ethical review.

Patient and public involvement Since this was an audit on an emergency switch, patients and public could not be involved in the beginning. However, feedback was taken from involved patients as described in the study.

\section{References}

1. Organization WH(2020) WHO Director-General's opening remarks at the media briefing on COVID-19-11 March 2020. Geneva, Switzerland

2. Anon. India's Third Coronavirus Patient Discharged From Hospital. NDTV.com. Available at: https://www.ndtv.com/indianews/covid-19-cases-indias-third-coronavirus-patient-dischargedfrom-hospital-2183009. Accessed March 23, 2020

3. Anon. Ministry of Health and Family Welfare | GOI RSS. Available at: https://www.mohfw.gov.in/. Accessed March 22, 2020

4. Anon. Kerala Population Sex Ratio in Kerala Literacy rate data 2011-2020. Available at: https://www.census2011.co.in/census/ state/kerala.html. Accessed March 22, 2020

5. Rahim AA, Chacko TV (2019) Nipah outbreak in North Kerala what worked? Insights for future response and recovery based on examination of various existing frameworks. Indian J Public Health 63:261-264

6. Joshi V (2019) Fellowship in rheumatology. Indian J Rheumatol $14: 250-250$

7. Anon. Countries with most WhatsApp users 2019 Statista. Available at: https://www.statista.com/statistics/289778/countrieswith-the-most-facebook-users/. Accessed March 25, 2020

8. Kandel N, Chungong S, Omaar A, Xing J (2020;0. Available at: https://www.thelancet.com/journals/lancet/article/PIIS01406736(20)30553-5/abstract. Accessed March 23, 2020) Health security capacities in the context of COVID-19 outbreak: an analysis of International Health Regulations annual report data from 182 countries. Lancet 395:1047-1053

9. Wang J, Xu C, Wong YK, He Y, Adegnika AA, Kremsner PG, Agnandji ST, Sall AA, Liang Z, Qiu C, Liao FL, Jiang T, Krishna S, Tu Y (2020;0. Available at: https://www.thelancet.com/journals/ lancet/article/PIIS0140-6736(20)30561-4/abstract. Accessed March 23, 2020) Preparedness is essential for malaria-endemic regions during the COVID-19 pandemic. Lancet 395:1094-1096

10. Ateriya N, Saraf A, Meshram VP, Setia P (2018) Telemedicine and virtual consultation: the Indian perspective. Natl Med J India 31: 215-218

11. Lurie N, Carr BG (2018) The role of telehealth in the medical response to disasters. JAMA Intern Med 178:745-746

12. Piga M, Cangemi I, Mathieu A, Cauli A (2017) Telemedicine for patients with rheumatic diseases: systematic review and proposal for research agenda. Semin Arthritis Rheum 47:121-128

13. Kataria S, Ravindran V (2018) Digital health: a new dimension in rheumatology patient care. Rheumatol Int 38:1949-1957 
14. Williams L, Rasmussen S, Kleczkowski A, Maharaj S, Cairns N (2015) Protection motivation theory and social distancing behaviour in response to a simulated infectious disease epidemic. Psychol Health Med 20:832-837

15. Porter G, Grills N (2016) Medication misuse in India: a major public health issue in India. J Public Health (Oxf) 38:e150-e157
16. Anon. techINSIGHT - At 502.2 million smartphone users, its time for smartphone brands to turn towards services in 2020 - techARC. Available at: https://techarc.net/techinsight-at-502-2-millionsmartphone-users-its-time-for-smartphone-brands-to-turn-towardsservices-in-2020/. Accessed March 25, 2020

Publisher's note Springer Nature remains neutral with regard to jurisdictional claims in published maps and institutional affiliations. 